\title{
Fuzzy Systems Based on Multispecies PSO Method in Spatial Analysis
}

\author{
Ferdinando Di Martino, ${ }^{1}$ Vincenzo Loia, ${ }^{2}$ and Salvatore Sessa ${ }^{1}$ \\ ${ }^{1}$ Dipartimento di Costruzioni e Metodi Matematici in Architettura, Università degli Studi di Napoli Federico II, \\ Via Monteoliveto 3, 80134 Napoli, Italy \\ ${ }^{2}$ Dipartimento di Matematica e Informaica, Università degli Studi di Salerno, Via Ponte don Melillo, 84084 Fisciano, Italy
}

Correspondence should be addressed to Salvatore Sessa, sessa@unina.it

Received 15 April 2012; Accepted 31 May 2012

Academic Editor: Irina G. Perfilieva

Copyright ( $) 2012$ Ferdinando Di Martino et al. This is an open access article distributed under the Creative Commons Attribution License, which permits unrestricted use, distribution, and reproduction in any medium, provided the original work is properly cited.

We present a method by using the hierarchical cluster-based Multispecies particle swarm optimization to generate a fuzzy system of Takagi-Sugeno-Kang type encapsulated in a geographical information system considered as environmental decision support for spatial analysis. We consider a spatial area partitioned in subzones: the data measured in each subzone are used to extract a fuzzy rule set of above mentioned type. We adopt a similarity index (greater than a specific threshold) for comparing fuzzy systems generated for adjacent subzones.

\section{Introduction}

A geographical information system (shortly, GIS) is often used by the analyst as a decision support system for problem solving in spatial analysis (e.g., cf. [1-9]). In some works, fuzzy inference systems are encapsulated in GIS tools, where the knowledge is represented by if-then rules. In [7], the authors present an inference model, integrated in a GIS, based on Takagi-Sugeno-Kang (shortly, TSK) fuzzy system $[10,11]$. In [4], a first-order Takagi-Sugeno fuzzy inference system is generated for estimating and simulating the discharge and sediment concentration in river basins. Also here we integrate in a tool GIS an inference model which generates a TSK-fuzzy system, based on the hierarchical clusterbased multiSpecies particle swarm optimization (shortly, HCMSPSO) algorithm [12] The spatial area of study is divided into subzones: for each subzone the correspondent TSK-fuzzy system is extracted by starting from the subset of patterns georeferred in that subzone. In our method, we calculate a similarity index between TSK-fuzzy systems generated for adjacent subzones: if this index is greater than a specific threshold, then the corresponding subzones and the data subsets are merged, and the TSK-fuzzy system is generated again for the new subzone. The partition of the area of study in subzones derives from the fact that the impact of the geographical characteristics necessarily involves spatial changes of the parameters forming the rule set of the fuzzy system.

A subzone represents a spatial subarea (of the area of study) with homogeneous characteristics with respect to the phenomenon investigated: we can consider as examples of subzones those areas with a specific index (pollution index, risk index, vulnerability index, traffic index, etc.). The concept of division of the area of study in homogeneous subzones has been introduced in [13-15], in which the area is partitioned in iso-reliable zones, that is, in subzones with homogeneous reliability of the spatial characteristics. Strictly speaking, the expert has provided a set of $N$ patterns $\left(x_{1}, \ldots, x_{n}, y\right)$, where $\mathbf{x}=\left(x_{1}, \ldots, x_{n}\right)$ is the $n$-dimensional vector input, and $y$ is the output, measured on georeferred locations in the area of study. This expert does not know a priori the optimal partition of the area of study in homogeneous subzones with respect to the fuzzy system to be generated; he already uses partitions defined for sociological, morphological, climatic characteristics. However, he does not know whether the same fuzzy system can be applied to 
two adjacent subzones. In this work, we propose a based particle swarm optimization (PSO) algorithm for optimizing the partition in homogeneous zones of the area of study: indeed we generate a TSK-fuzzy system for each subzone by using the so-called HCMSPSO algorithm [12], which is a variation of PSO, and we compare the TSK-fuzzy system in adjacent subzones. A TSK-fuzzy system [16] is composed from a set of $r$ fuzzy rules $R_{i}$ represented in the following form:

$$
\begin{array}{r}
R_{i}: \operatorname{IF}\left(x_{1} \text { is } A_{i 1}\right) \cdots\left(x_{n} \text { is } A_{i n}\right), \quad \text { then }\left(y \text { is } f_{i}\right), \\
i=1, \ldots, r,
\end{array}
$$

where the fuzzy set $A_{i j}$ assumes the following Gaussian membership function:

$$
A_{i j}=\exp \left[-\left(\frac{x_{j}-m_{i j}}{\sigma_{i j}}\right)^{2}\right]
$$

The firing strength of the $i$ th rule on $\mathbf{x}=\left(x_{1}, \ldots, x_{n}\right)$ is given as

$$
\mu_{i}(\mathbf{x})=\prod_{j=1}^{n} A_{i j}=\exp \left[-\sum_{j=1}^{n}\left(\frac{x_{j}-m_{i j}}{\sigma_{i j}}\right)^{2}\right] .
$$

In the TSK-fuzzy system of zero order, the fuzzy set $f_{i}$ is generally represented in the consequent as a constant $a_{i 0}$; the TSK-fuzzy system of one order is given by a linear combination of the input variables:

$$
f_{i}=a_{i 0}+\sum_{i=1}^{n} a_{i j} x_{j}
$$

where the coefficient $a_{i j}$ are real numbers. The output $y$ is calculated by the weighted average defuzzification method:

$$
y^{\prime}=\frac{\sum_{i=1}^{r} \mu_{i}(\mathbf{x}) f_{i}}{\sum_{i=1}^{r} \mu_{i}(\mathbf{x})} .
$$

The HCMSPSO algorithm generates an optimal TSKfuzzy system by determining the number $r$ of rules and by optimizing the coefficient values $m_{i j}, \sigma_{i j}$, and $a_{i j}$ in each rule. In our approach, the geographical area of study is initially partitioned in $Z$ subzones; for each subzone we apply the HCMSPSO algorithm to generate the optimal TSKfuzzy system. Here, we give an evolution process in which at each iteration we merge adjacent subzones with a fuzzy rule-set similarity index greater then a predefined threshold. The process stops when no fuzzy rule-set similarity index associated to couple of adjacent subzones is greater or equal to the threshold. We propose a similarity index based on the fuzzy inclusion concept given as

$$
I_{i i^{\prime} j}^{h k}=\frac{\int_{x_{j 0}}^{x_{j 1}} \min \left[A_{i j}^{h}\left(x_{j}\right), A_{i^{\prime} j}^{k}\left(x_{j}\right)\right] d x_{j}}{\max \left[\int_{x_{j 0}}^{x_{j 1}} A_{i j}^{h}\left(x_{j}\right) d x_{j}, \int_{x_{j 0}}^{x_{j 1}} A_{i^{\prime} j}^{k}\left(x_{j}\right) d x_{j}\right]},
$$

where $x_{j 0}$ and $x_{j 1}$ are the lower and upper bounds of the domain of $x_{j}$. If $I_{i j}^{h k}=1$, the two fuzzy sets overlap completely.
In other words, given the $h$ th and $k$ th TSK-fuzzy systems correspondent to adjacent subzones and with the same number of rules, we define the following similarity index for each couple of rules $r_{i}$ and $r_{i^{\prime}},\left(i, i^{\prime}\right) \in(1, \ldots, r)^{2}$ as

$$
I_{i i^{\prime}}^{h k}=\frac{\sum_{j=1}^{n+1} I_{i i^{\prime} j}^{h k}}{n+1}
$$

which is a mean of (6) on the $n+1$ variables ( $n$ inputs and the output variable). Then, we order the rule sets of the two fuzzy systems in accordance to the following criteria:

(i) we choose the two rules $r_{i}$ and $r_{i^{\prime}}$ with the greatest value $I_{i i}^{h k}$. The two rules become the first rules of the respective fuzzy systems, and the index (7) can be written as $I_{1}^{h k}$;

(ii) among the remaining rules, we consider the two new rules with the index (7); these rules become the second rules of the respective fuzzy systems and the index (7) can be written as $I_{2}^{h k}$; the process is repeated up to order all the rules in the two fuzzy sets.

We calculate the similarity index of the two TSK-fuzzy systems as

$$
S^{h k}=\frac{\sum_{i=1}^{r} I_{i}^{h k}}{r} .
$$

The similarity value $S^{h k}$ is a mean of the values $I_{i}^{h k}$ obtained for all the rules. We use this index to decide if the $h$ th and $k$ th adjacent subzones can be merged. Our evolution process is a hierarchical iterative approach; initially the area of study is partitioned in a fine-grained set of subzones; the analyst divides the area of study into subzones based on significant geospatial characteristics with respect to the problem studied (e.g., he divides the area into subzones corresponding to municipalities in demographic problems, or corresponding to subzones with different climatic characteristics in meteorological problems). Since each subzone must contain a significant number of data points (otherwise the partition would be too fine with respect to the distribution of the patterns), we impose the constraint that each subzone must contain at least $N_{\text {th }}$ patterns, where $N_{\text {th }}$ stands for a threshold number. At each iteration, the HCMSPSO algorithm is applied to generate an optimal fuzzy system for each subzone. In Section 2, we introduce the HCMSPSO method, in Section 3 we present our method for finding the optimal partition of the area of study, in Section 4 we present some experimental results, and Section 5 is conclusive.

\section{The HCMSPSO Algorithm: An Overview}

The HCMSPSO algorithm [12] is a method based on the PSO algorithm for determining the optimal TSK-fuzzy system by using a set of patterns. The HCMSPSO determines the number $r$ of rules and the optimal values of the parameters $m_{i j}, \sigma_{i j}$, and $a_{i j}$ of the membership functions in each rule. In $[12,17-27]$, variations of the PSO algorithm are proposed. 
The HCMSPSO method originates from the clusterbased particle swarm optimization method (shortly, CPSO) [28], in which each swarm is used independently for optimizing a set of parameters. In HCMSPSO, each swarm forms a species and the number of species is set to the number of fuzzy rules $r$. each species is formed from $P_{s}$ particles, and each particle in a species represents a single fuzzy rule. The $i$ th species is used for optimizing the parameters in the $i$ th fuzzy rule. The position $s_{i}^{q}$ of the $q$ th particle in the $i$ th species is given from the $(2 n+1)$-dimensional vector:

$$
\mathbf{s}_{i}^{q}=\left(m_{i 1}^{q}, \ldots, m_{i n}^{q}, \sigma_{i 1}^{q}, \ldots, \sigma_{i n}^{q}, a_{i 0}^{q}\right) \in \mathfrak{R}^{2 n+1}
$$

and from the $(3 n+1)$-dimensional vector:

$$
\mathbf{s}_{i}^{q}=\left(m_{i 1}^{q}, \ldots, m_{i n}^{q}, \sigma_{i 1}^{q}, \ldots, \sigma_{i n}^{q}, a_{i 0}^{q}, a_{i 1}^{q}, \ldots, a_{i n}^{q}\right) \in \mathfrak{R}^{3 n+1} .
$$

for a TSK-fuzzy system of first order. A TSK-fuzzy system of zero order is built by choosing one for each species of the $P_{s}$ particles. To determine the final number of rules and generate the $P_{s}$ particles in each species, the following iterative process is used:

(1) initially we set $r=1$; we consider the first input pattern $\mathbf{x}(0)$, forming the first particle in the first species setting $m_{1 j}^{1}=x_{j}(0)$ and $\sigma_{1 j}^{1}=\sigma_{\text {Init }}$, where $\sigma_{\text {Init }}$ is a predefined value by determining the initial width of each fuzzy set;

(2) we generate all the particles in the first species, associated to the fuzzy rule $R_{1}$; the $q$ th particle is given by the formula (for TSK-fuzzy system of first order):

$$
\begin{aligned}
\mathbf{s}_{1}^{q}=( & m_{11}+\Delta m_{11}^{q}, \ldots, m_{i n}+\Delta m_{1 n}^{q}, \\
& \left.\sigma_{11}+\Delta \sigma_{11}^{q}, \ldots, \sigma_{1 n}+\Delta \sigma_{1 n}^{q}, a_{10}^{q}, a_{11}^{q}, \ldots, a_{1 n}^{q}\right),
\end{aligned}
$$

where $q=1, \ldots, P_{s}, \Delta m_{1 j}^{q}$ and $\Delta \sigma_{1 j}^{q}$ represent small variations to $m_{1 j}$ and $\sigma_{1 j}$, respectively, generated from the interval $[-0.1,0.1]$; the values $a_{10}^{q}, a_{11}^{q}, \ldots, a_{1 n}^{q}$ are obtained randomly in the output $y$ range;

(3) for each successive pattern $\mathbf{x}(k), k=2, \ldots, N$, we consider the rule with maximum firing strength given by

$$
I(k)=\arg \max _{1 \leq i \leq r}\left[\mu_{i}(\mathbf{x}(k))\right] .
$$

If results $\mu_{\mathrm{I}(k)}(\mathbf{x}(k)) \leq \mu_{\mathrm{th}}$, where $\mu_{\mathrm{th}}$ is a predefined threshold, then a new rule is generated by setting

$$
\begin{gathered}
m_{(r+1) j}(k)=x_{j}(k), \quad j=1, \ldots, n, \\
\sigma_{(r+1) j}(k)=\alpha \sum_{j=1}^{n}\left(\frac{x_{j}(k)-m_{I_{k} j}}{\sigma_{I_{k} j}}\right)^{2}, \quad j=1, \ldots, n,
\end{gathered}
$$

where $\alpha>0$ determines the degree of overlapping between two clusters;
(4) we generate all the particles in the $(r+1)$-species, associated to the fuzzy rule $R_{(r+1)}$; the $q$ th particle is given by the formula (for TSK-fuzzy system of first order):

$$
\begin{aligned}
& \mathbf{s}_{(r+1)}^{q}(k) \\
& =\left(m_{(r+1) 1}(k)+\Delta m_{(r+1) 1}^{q}(k), \ldots, m_{(r+1) n}(k)\right. \\
& \quad+\Delta m_{(r+1) n}^{q}(k), \sigma_{(r+1) 1}(k)+\Delta \sigma_{(r+1) 1}^{q}(k), \ldots, \sigma_{(r+1) n}(k) \\
& \left.\quad+\Delta \sigma_{(r+1) n}^{q}(k), a_{(r+1) 0}^{q}, a_{(r+1) 1}^{q}, \ldots, a_{(r+1) n}^{q}\right),
\end{aligned}
$$

where $q=1, \ldots, P_{s}, k=2, \ldots, N, \Delta m_{(r+1) j}^{q}(k)$ and $\Delta \sigma_{(r+1) j}^{q}(k)$ represent small variations to $m_{(r+1) j}(k)$ and $\sigma_{(r+1) j}(k)$, respectively, from the interval $[-0.1,0.1]$; the related values $a_{(r+1) 0}^{q}, a_{(r+1) 1}^{q}, \ldots, a_{(r+1) n}^{q}$ are obtained randomly from an interval identical to the output $y$ range;

(5) we set $r=r+1$ and iterate the steps (3) and (4) for all the patterns. At the end of the process, we have generated $r$ rules and $r$ species with $P_{s}$ particles.

For each species, a partition of its particles is created in subspecies. A subspecies is a cluster of particles of a species. To create a subspecies partition of the particle of a species, we need to sort the particle of the species. The index used for sorting the particles of a species is the root means square error (RMSE) defined as

$$
\operatorname{RMSE}=\sqrt{\frac{\sum_{k=1}^{N}\left[y^{\prime}(k)-y(k)\right]^{2}}{N}},
$$

where $y^{\prime}(k)$ is the output calculated using the defuzzification formula (5), and $y(k)$ is the output value of the $k$ th pattern. The successive steps are used for sorting the particles in each species based on the increasing value of the RMSE and partitioning each species into subspecies:

(1) for each combination of particles of each species, we determine the set of particles $\left(\boldsymbol{s}_{1}^{g}, \ldots, \boldsymbol{s}_{r}^{g}\right)$ with minimum RMSE. For each species, we set $s_{i}^{1}=s_{i}^{g}$;

(2) for sorting the particles in the $i$ th species, we calculate the RMSE produced by the combination $\left(\mathbf{s}_{1}^{g}, \ldots, \mathbf{s}_{i-1}^{g}, \mathbf{s}_{i}^{q}, \mathbf{s}_{i+1}^{g}, \ldots, \mathbf{s}_{r}^{g}\right)$ with $q=2, \ldots, P_{s}$; then sorting the particles based on the increasing value of the corresponding RMSE. This step is repeated for all $r$ species.

After sorting each species, we can do a partition of them into subspecies; the first particle of a subspecies, that is, the particle of the subspecies with least RMSE, is called leader of the subspecies. The next steps are used for partitioning each ordered species in subspecies:

(1) for the $i$ th species, we set the number of subspecies $n_{i}=1$ and create the first subspecies by setting the leader of this subspecies as the first particles of the species, $\mathbf{p}_{i}^{\operatorname{leader}\left(n_{i}\right)}=$ $\mathbf{s}_{i}^{g}$;

(2) then we consider the successive particles of the species $s_{i}^{q}$ with $q=2, \ldots, P_{s}$ and calculate the index distance between the particle $s_{i}^{q}$ and the leader of the first subspecies:

$$
\psi\left(\mathbf{p}_{i}^{\operatorname{leader}\left(n_{i}\right)}, \mathbf{s}_{i}^{q}\right)=\sqrt{\frac{\left\|\mathbf{p}_{i}^{\text {leader }\left(n_{i}\right)}-\mathbf{s}_{i}^{q}\right\|}{L}},
$$


TABle 1: Dimension of the pattern subsets included in each subzone.

\begin{tabular}{lcc}
\hline Subzone & Class & Number of buildings \\
\hline 1 & Industrial area & 64 \\
2 & Industrial area & 75 \\
3 & Rural area & 1306 \\
4 & Inhabited nucleus & 586 \\
5 & Inhabited nucleus & 126 \\
6 & Urban center & 192 \\
7 & Rural area & 1160 \\
\hline
\end{tabular}

where $L=2 n$ (resp., $L=3 n$ ) for a TSK-fuzzy system of zero (resp., first) order. If we have $\psi\left(\mathbf{p}_{i}^{\operatorname{leader}\left(n_{i}\right)}, \mathbf{s}_{i}^{q}\right)<\psi$ th, where $\psi_{\text {th }}$ is a threshold value, then we assign the particle $s_{i}^{q}$ to this subspecies; otherwise we create a new subspecies by setting $n_{i}=n_{i}+1$

(3) we iterate the steps (8) and (9) for all the $r$ species.

In the last steps, the PSO algorithm is applied; we define the particles in the same subspecies as neighbours of a particle. The best global position of the neighbours of a particle $s_{i}^{q}$, enclosed in the $k$ th subspecies of the $i$ th species at the iteration time $(t-1)$, is given from the best position of the leader of the subspecies $\mathbf{p}_{i}^{\text {leader }(k)}$ until the iteration time $(t-1)$. The best local position of the particle $\mathbf{s}_{i}^{q}$ enclosed in the $k$ th subspecies of the $i$ th species at the iteration time $(t-1)$ is given by the best position $\mathbf{p}_{i}^{q}$ of the particle until the iteration time $(t-1)$.

\section{Subzones in the Generation Process}

Our method is an iterative process that determines the optimal partition in subzones of the area of study; each subzone represents a zone of the area of study, homogeneous with respect to a specific TSK-fuzzy system composed from $r$ fuzzy rules in the form (1). The expert creates an initial finer partition of the area of study according to specific local features (of type sociological, climatic, orographic, hydrological, etc.); the pattern data set is divided into subsets such that a subset is spatially included in the corresponding subzone. The successive step is performed to verify that the data distribution is consistent with the partition into subzones of the area of study; we verify that the number of patterns inside each subzone is greater or equal to a specific threshold value which can be set by the user. Clearly, the higher this value is, more the low resultant RMSE is expected; therefore, the greater the accuracy of the resulting TSK-fuzzy system will be. If $N_{h}$ is the dimension of the subset of patterns inside the $h$ th subzone and $N_{\text {th }}$ is the threshold value, we impose the following constraint for each subzone:

$$
N_{h} \geq N_{\text {th }}, \quad h=1, \ldots, Z,
$$

where $Z$ is the cardinality of the partition such that $N=$ $\sum_{h=1}^{Z} N_{h}$. We consider consistently the pattern dataset with the partition into subzones of the area of study if (17) is true; otherwise, the expert creates a more coarse-grained partition; this control is iterated until each subset of patterns is consistent with respect to the corresponding subzone. For each subzone, the HCMSPSO method is applied for generating an optimal TSK-fuzzy system; we associate to each subzone the TSK-fuzzy system determined and its RMSE.

We compare the TSK-fuzzy systems of adjacent subzones, calculating the similarity index $S^{h k}$ as in (8). If $S^{h k}$ is greater or equal to a threshold value $S^{\text {threshold, }}$ the $h$ th and $k$ th subzones are merged. When two or more subzones are merged in a new subzone, we group the corresponding pattern subsets together into a single subset, and we restart the HCMSPSO algorithm for the new subzone. This process is iterated until we have that $S^{h k}<S^{\text {threshold }}$ for all $h$ th and $k$ th adjacent subzones. As final result a thematic map is produced in which the area of study is divided into the final subzones classified according to the RMSE of the TSK-fuzzy system generated. To compare homogeneous errors, we calculate two normalized errors used in the literature:

(i) the normalized root mean square Error index (shortly, NRMSE) which is the rapport between the RMSE and the range (this last is given from the difference between maximal and the minimal values of the output variable $y$, in absolute value). We define the NRMSE with the following percentage:

$$
\text { NRMSE }=\frac{\text { RMSE }}{\left|y_{\max }-y_{\min }\right|} \times 100 ;
$$

(ii) the coefficient of variation of the RMSE error (shortly, CVRMSE) which is the rapport between the RMSE and the mean value $\bar{y}$ of the output variable $y$. We define the CVRMSE with the following percentage:

$$
\text { CVRMSE }=\frac{\text { RMSE }}{\bar{y}} \times 100 .
$$

The NRMSE and CVRMSE are used in the creation of thematic maps of the TSK-fuzzy systems. The expert can fix a reliability threshold for the TSK-fuzzy system; in subzones with index greater than this threshold, it is necessary to use additional data and/or eliminate data with noise and outliers. The process described above can be schematized in the following steps:

(1) the expert divides initially the area of study into $\mathrm{Z}$ subzones, partitioning the area in homogeneous zones; this partition represents the finer partition desired by the expert;

(2) the pattern dataset is partitioned in subsets of data; each subset contains measured data georeferred into a specific subzone; if the dimension of each pattern subsets is less than a prefixed threshold $N_{\text {th }}$, the partition is too fine with respect to the pattern dataset and the process return to step (1); in this case the expert must create a more coarse grained subzone partition of the area of study;

(3) for each subzone, we use the HCMSPSO method to determine the number of rules and generate the correspondent species;

(4) we use the HCMSPSO method to generate the subspecies and optimize the parameters in each rule; 


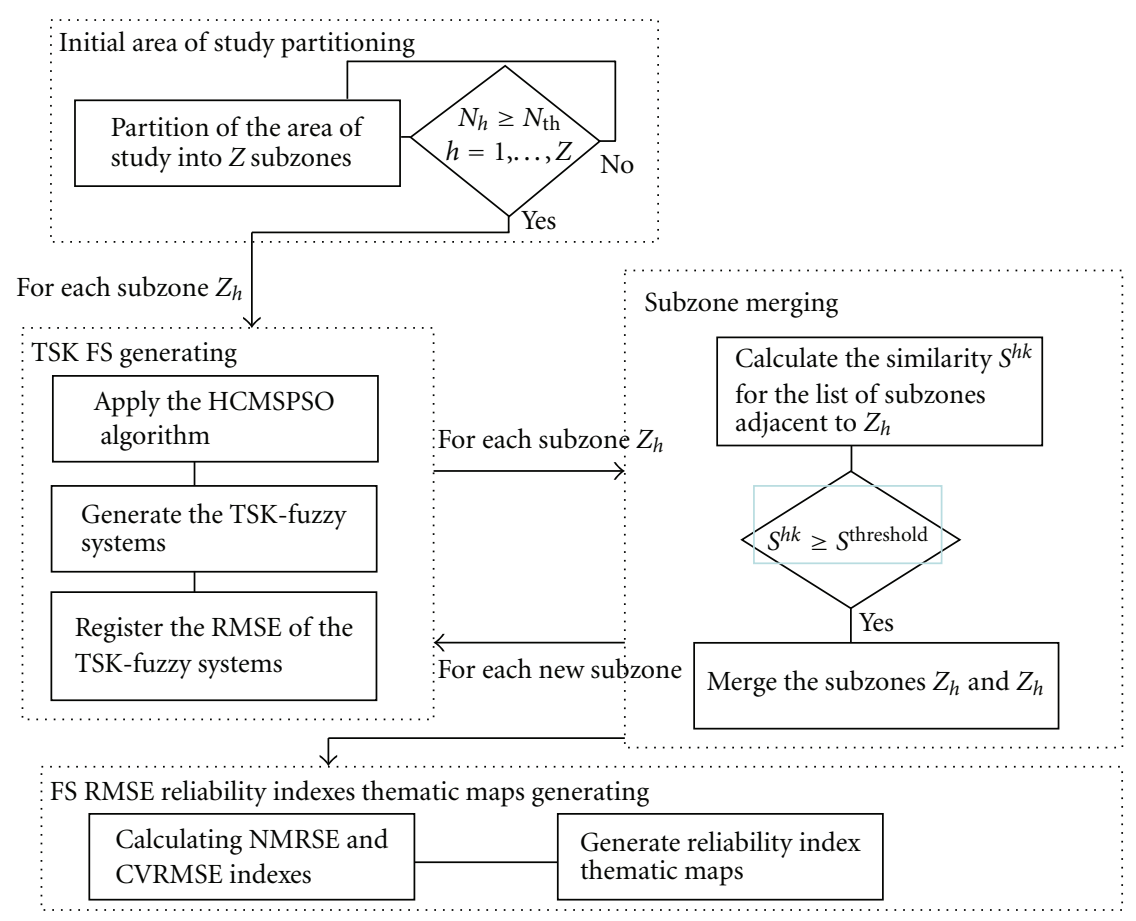

FIGURE 1: Schema of the subzones generation process.

TABLE 2: Final number of rules and RMSE obtained for each TSK-fuzzy system.

\begin{tabular}{lccccc}
\hline Subzone & Number of rules & RMSE & NRMSE & CVRMSE & $t_{p}$ \\
\hline 1 & 7 & 15.281 & $2.942 \%$ & $5.736 \%$ & $1.2 \times 10^{5}$ \\
2 & 7 & 22.214 & $4.738 \%$ & $8.175 \%$ & $1.3 \times 10^{5}$ \\
3 & 239.023 & $45.020 \%$ & $83.677 \%$ & $1.5 \times 10^{5}$ \\
4 & 87.727 & $18.465 \%$ & $32.460 \%$ & $1.3 \times 10^{5}$ \\
5 & 7 & 38.369 & $9.820 \%$ & $14.518 \%$ & $1.4 \times 10^{5}$ \\
6 & 7 & 53.223 & $10.467 \%$ & $18.835 \%$ & $1.4 \times 10^{5}$ \\
7 & 7 & 162.931 & $30.688 \%$ & $57.169 \%$ & $1.5 \times 10^{5}$ \\
\hline
\end{tabular}
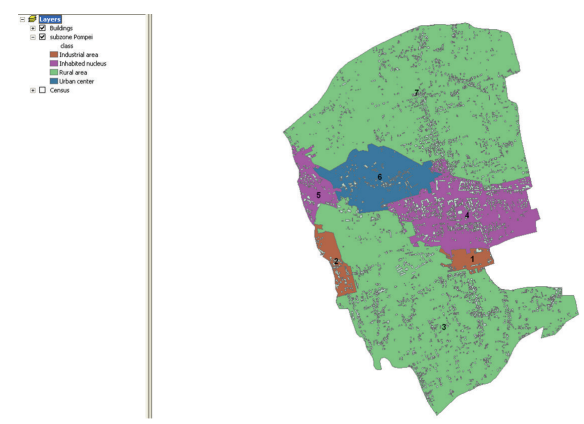

Figure 2: Initial subzones of Pompeii based on the city planning class.

(5) we compare the TSK-fuzzy system calculated to two adjacent subzones $z_{h}$ and $z_{k}$, by calculating the similarity index $S^{h k}$; if $S^{h k} \geq S^{\text {threshold }}$, where $S^{\text {threshold }}$ is a predefined

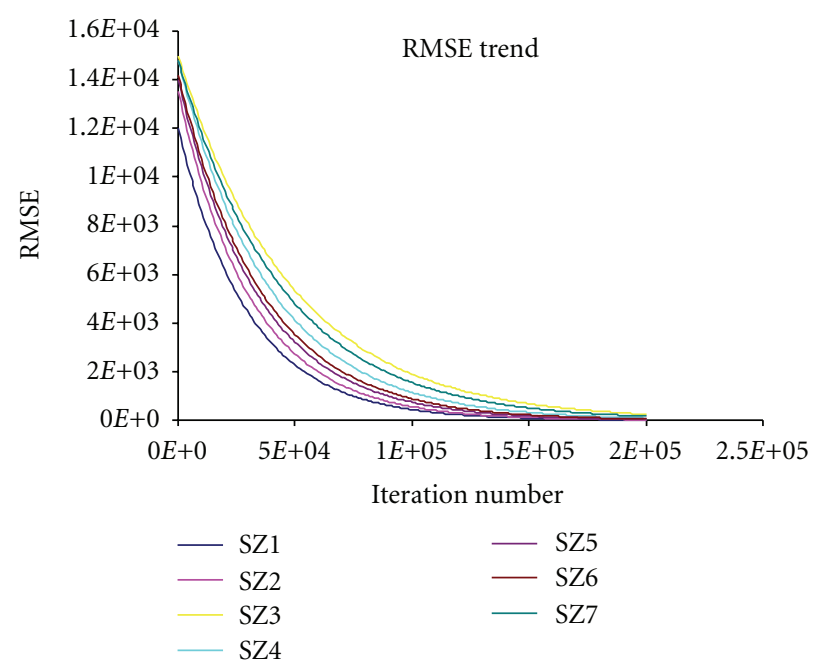

Figure 3: RMSE trend obtained for the seven subzones. 
TABLE 3: Similarity index between adjacent subzones.

\begin{tabular}{lcc}
\hline Subzone 1 & Subzone 2 & $S^{h k}$ \\
\hline 4 & 6 & 0.388 \\
5 & 6 & 0.326 \\
1 & 4 & 0.078 \\
2 & 5 & 0.073 \\
\hline
\end{tabular}

TABLE 4: Breaks of the three thematic classes of NRMSE and CVRMSE.

\begin{tabular}{lcc}
\hline Class & NRMSE class breaks & CVRMSE class breaks \\
\hline Low & $0 \%-10 \%$ & $0 \%-20 \%$ \\
Mean & $10,001 \%-30 \%$ & $20,001 \%-50 \%$ \\
High & Over $30 \%$ & Over $50 \%$ \\
\hline
\end{tabular}

threshold, the two subzones, are merged in one subzone and the number of subzones is $Z-1$;

(6) if there are two or more subzones merged, we iterate steps (3), (4), and (5) for each new subzone;

(7) two thematic maps are created by showing for each final subzone the reliability class correspondent to the final NRMSE and CVRMSE.

In Figure 1, we have schematized the above process. If we are interested to analyze whether the final distribution of the final subzones is or not approximately uniform, we can calculate the coefficient of variation of this distribution; this index is extracted by calculating the mean and standard deviation of the distribution of the $N_{h}$ 's as

$$
\begin{gathered}
m_{N}=\frac{\sum_{h=1}^{Z} N_{h}}{Z}, \\
\sigma_{N}=\sqrt{\frac{\sum_{h=1}^{Z}\left(N_{h}-m_{N}\right)^{2}}{Z-1} .} .
\end{gathered}
$$

The coefficient of variation, expressed in percentage, is given by

$$
\mathrm{CV}_{N}=\frac{\sigma_{N}}{m_{N}} \times 100
$$

We can consider the pattern data distribution approximately uniform if $\mathrm{CV}_{N} \leq 50 \%$; this control can be used at the end of the process by the expert if he intends to verify if the final pattern dataset is approximately uniform with respect to the final partition of the area of study. This analysis is useful to see that significant differences in the RMSE on the area of study can be due to substantial changes in the cardinality of the final subsets of patterns inside each final subzone. In Section 4, we present some results of our tests applied on spatial datasets. The HCMSPSO algorithm has been implemented and encapsulated in the tool GIS ESRI/ArcGIS, release 10. A first experiment is made on test spatial data firstly by comparing the results obtained with the HCMSPSO method and the ones obtained using other PSO-based methods, secondly for verifying the accuracy of our method in the subzones merging process. Then, we present the results obtained by applying our method on a

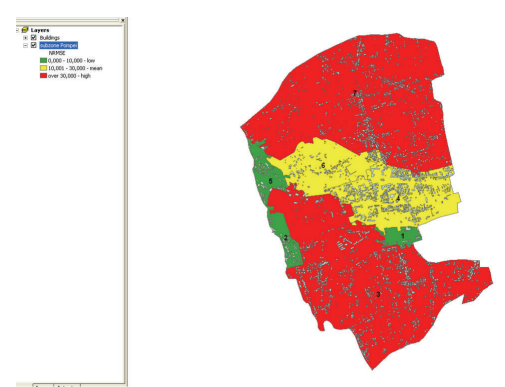

FIgURE 4: Thematic map of NRMSE.

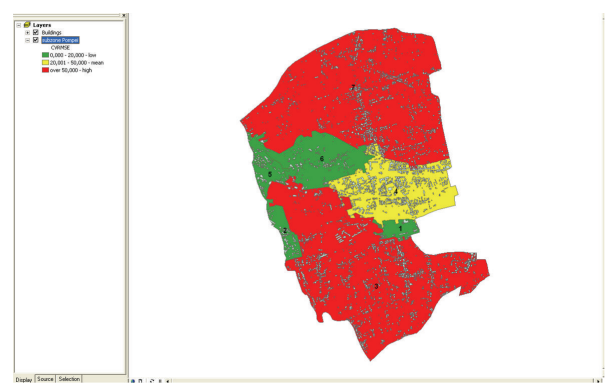

FIgURE 5: Thematic map of CVRMSE.

problematic related to the valuation of the costs for building maintenance in the city of Pompeii (Italy).

\section{Results of Tests}

Now, we apply our method to geospatial data. The test concerns the buildings of the municipality of Pompeii, which is a famous touristic city for the presence of an important Sanctuary and of a large famous archeological heritage. The municipal area is partitioned into four classes: rural area, urban center, inhabited nucleus, and industrial area. An expert's goal is to plan the cost of maintenance of buildings, based to the building maintenance data. The data are extracted from the related dataset, and they are the following: $x_{1}$ : year of construction, $x_{2}$ : year of last maintenance, $x_{3}$ : extent of the damage with respect to the volume of the building $(0:$ null extension and 1 : complete extension), $x_{4}$ : gravity of the damage $(0$ : null gravity and 1 : maximum gravity), $y$ : cost of the maintenance (calculated in thousands of Euro). Each pattern corresponds to a building georeferred to a specific census microzone. The initial subzones are formed by union of adjacent microzones with a same city planning class, and we obtain $Z=7$ subzones. The thematic map in Figure 2 shows the seven subzones obtained by the subdivision of the area of Pompeii based on the four classes.

The dataset is formed by $N=3445$ buildings (cf. Table 1); we set $N_{\text {th }}=50$. We have that $N_{h}$ is greater than $N_{\text {th }}=50$ for every $h=1, \ldots, Z$, we can assume that the set of data is suitable for the partition. Then we use this subdivision in 7 subzones of Pompeii for generating the relative TSKfuzzy system of zero order. In the HCMSPSO method, we set 
the threshold values $\mu_{\text {th }}$ and $\psi_{\text {th }}$ to 0.03 and 2, respectively; the number of iterations is 200.000. The graph in Figure 3 shows the RMSE trend with respect to the iteration number obtained for the seven subzones. For all the seven TSK-fuzzy systems generated, the RMSE trend reaches a plateau after about $1.5 \times 10^{5}$ iterations. Table 2 shows the results, and we report the final number of rules, the RMSE, NRMSE, CVRMSE, and the index $t_{p}$. The results show that the TSKfuzzy systems related to the subzones 3 and 7 have RMSE and $t_{p}$ greater values with respect to the ones related to the other subzones. Then, we calculated the similarity indexes between adjacent subzones; we set $S^{\text {threshold }}=0.7$.

The similarity values between two TSK-fuzzy systems with same number of rules, associated with adjacent subzones are given in Table 3. Table 4 contains breaks of the three thematic classes of NRMSE and CVRMSE: we consider not sufficiently reliable the results obtained in subzones belonging to the class "high," that is, subzones with NMRSE greater then $30 \%$ or CVRMSE greater than $50 \%$. The results in Figures 4 and 5 show that the resultant TSK-fuzzy system obtained for the rural areas 1 and 7 are not sufficiently reliable. These subzones contain the greatest number of patterns; the results confirm that the costs of maintenance of a building in rural areas are probably not significantly related to parameters like the last year of construction and maintenance.

\section{Conclusions}

In this paper, we present a method based on the HCMSPSO algorithm for extracting a TSK-fuzzy system from a dataset of georeferred measured data for spatial analysis. The area of study is partitioned initially into subzones by the expert; for each subzone is extracted the TSK-fuzzy system by using the subset of patterns georeferred into the subzone. We merge subzones with similar TSK-fuzzy system and by recreating the TSK-fuzzy system for the new subzone. For each TSKfuzzy system, the reliability is evaluated using indexes based on the final RMSE. The algorithm has been implemented in the tool ESRI/ArcGIS, release 10; the results of our tests show that this method can be well used in a GIS platform and encapsulated as decision support system for optimizing fuzzy systems related to subzones of the area of study.

\section{References}

[1] O. Al-Jarrah and H. Abu-Qdais, "Municipal solid waste landfill siting using intelligent system," Waste Management, vol. 26, no. 3, pp. 299-306, 2006.

[2] A. Ertug Gunes and J. P. Kovel, "Using GIS in emergency management operations," Journal of Urban Planning and Development, vol. 126, no. 3, pp. 136-149, 2000.

[3] P. Kumar, V. Singh, and D. Reddy, "Advanced traveler information system for Hyderabad City," IEEE Transactions on Intelligent Transportation Systems, vol. 6, no. 1, pp. 26-37, 2005.

[4] P. C. Nayak and S. K. Jain, "Modelling runoff and sediment rate using a neuro-fuzzy technique," Proceedings of the Institution of Civil Engineers, vol. 164, no. 4, pp. 201-209, 2011.
[5] E. V. Ocalir, O. Y. Ercoskun, and R. Tur, "An integrated model of GIS and fuzzy logic (FMOTS) for location decisions of taxicab stands," Expert Systems with Applications, vol. 37, no. 7, pp. 4892-4901, 2010.

[6] A. P. Tang, L. L. Xie, and X. X. Tao, "Application of GIS to build earthquake emergency response system for urban area," Journal of Harbin Institute of Technology, vol. 9, no. 1, pp. 3842, 2002.

[7] C. H. Tsai, W. C. Wang, C. W. Chen, H. L. Chen, and M. L. Lin, "The construction of decision model for tourism disaster evacuation based on GIS and fuzzy theory," World Academy of Science, Engineering and Technology, vol. 54, pp. 81-85, 2009.

[8] M. H. Vahidnia, A. A. Alesheikh, and A. Alimohammadi, "Hospital site selection using fuzzy AHP and its derivatives," Journal of Environmental Management, vol. 90, no. 10, pp. 3048-3056, 2009.

[9] D. Zheng and and W. Kainz, "Fuzzy rule extraction from GIS data with a neural fuzzy system for decision making," in Proceedings of the 7th International Symposium on Advances in Geographic Information Systems (ACM-GIS '99), pp. 79-84, Kansas, Kan, USA, November 1999.

[10] M. Sugeno and G. T. Kang, "Structure identification of fuzzy model," Fuzzy Sets and Systems, vol. 28, no. 1, pp. 15-33, 1988.

[11] T. Takagi and M. Sugeno, "Fuzzy identification of systems and its applications to modeling and control," IEEE Transactions on Systems, Man and Cybernetics, vol. 15, no. 1, pp. 116-132, 1985.

[12] C. F. Juang, C. M. Hsiao, and C. H. Hsu, "Hierarchical clusterbased multispecies particle-swarm optimization for fuzzysystem optimization," IEEE Transactions on Fuzzy Systems, vol. 18, no. 1, pp. 14-26, 2010.

[13] F. Di Martino, S. Sessa, and V. Loia, "A fuzzy-based tool for modelization and analysis of the vulnerability of aquifers: a case study," International Journal of Approximate Reasoning, vol. 38, no. 1, pp. 99-111, 2005.

[14] F. Di Martino, V. Loia, S. Sessa, and M. Giordano, "An evaluation of the reliability of a GIS based on fuzzy logic, in a concrete case study," in Fuzzy Modeling with Spatial Information for Geographic Problems, F. E. Petry, V. B. Robinson, and M. A. Cobb, Eds., pp. 185-208, Springer, Heidelberg, Germany, 2005.

[15] F. Di Martino, V. Loia, S. Sessa, and S. B, "By using fuzzy rules for applications to risk analysis in the Vesuvian area," Contemporary Engineering Sciences, vol. 4, no. 5, pp. 55-78, 2011.

[16] D. Kukolj, "Design of adaptive Takagi-Sugeno-Kang fuzzy models," Applied Soft Computing Journal, vol. 2, no. 2, pp. 89103, 2002.

[17] P. Bajpai and S. N. Singh, "Fuzzy adaptive particle swarm optimization for bidding strategy in uniform price spot market," IEEE Transactions on Power Systems, vol. 22, no. 4, pp. 2152-2160, 2007.

[18] D.-B Chen, F. Zou, and Y.-T. Wang, "A multi-objective endocrine PSO algorithm and application," Applied Soft Computing, vol. 11, no. 8, pp. 4508-4520, 2011.

[19] X. Chen and Y. Li, "A modified PSO structure resulting in high exploration ability with convergence guaranteed," IEEE Transactions on Systems, Man, and Cybernetics B, vol. 37, no. 5, pp. 1271-1289, 2007.

[20] C. F. Juang, "A hybrid of genetic algorithm and particle swarm optimization for recurrent network design," IEEE Transactions on Systems, Man, and Cybernetics B, vol. 34, no. 2, pp. $997-$ 1006, 2004. 
[21] D. Parrott and X. Li, "Locating and tracking multiple dynamic optima by a particle swarm model using speciation," IEEE Transactions on Evolutionary Computation, vol. 10, no. 4, pp. 440-458, 2006.

[22] K. E. Parsopóulos and M. N. Vrahatis, "Particle swarm optimization method in multiobjective problems," in Proceedings of ACM Symposium on Applied Computing, pp. 603-607, Madrid, Spain, March 2002.

[23] A. Ratnaweera, S. K. Halgamuge, and H. C. Watson, "Selforganizing hierarchical particle swarm optimizer with timevarying acceleration coefficients," IEEE Transactions on Evolutionary Computation, vol. 8, no. 3, pp. 240-255, 2004.

[24] Y. Shi and R. Eberhart, "Modified particle swarm optimizer," in Proceedings of the IEEE International Conference on Evolutionary Computation (ICEC' '98), pp. 69-73, May 1998.

[25] W. Jingxuan and W. Yuping, "Multi-objective fuzzy particle swarm optimization based on elite archiving and its convergence," Journal of Systems Engineering and Electronics, vol. 19, no. 5, pp. 1035-1040, 2008.

[26] Z. Xinchao, "A perturbed particle swarm algorithm for numerical optimization," Applied Soft Computing Journal, vol. 10, no. 1, pp. 119-124, 2010.

[27] W. Zhang and Y. Liu, "Multi-objective reactive power and voltage control based on fuzzy optimization strategy and fuzzy adaptive particle swarm," International Journal of Electrical Power and Energy Systems, vol. 30, no. 9, pp. 525-532, 2008.

[28] F. van den Bergh and A. P. Engelbrecht, "A cooperative approach to participle swam optimization," IEEE Transactions on Evolutionary Computation, vol. 8, no. 3, pp. 225-239, 2004. 

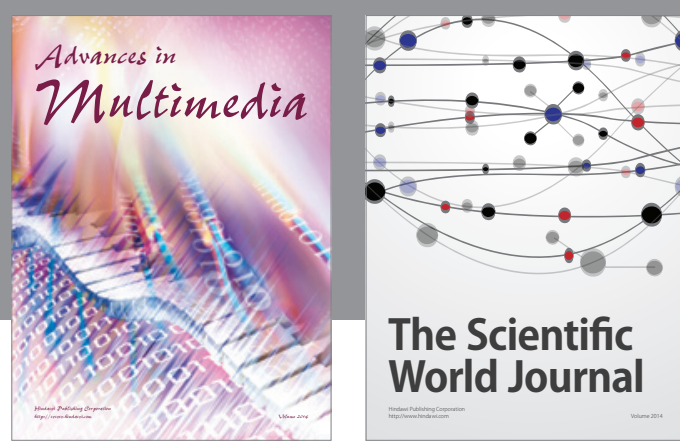

The Scientific World Journal
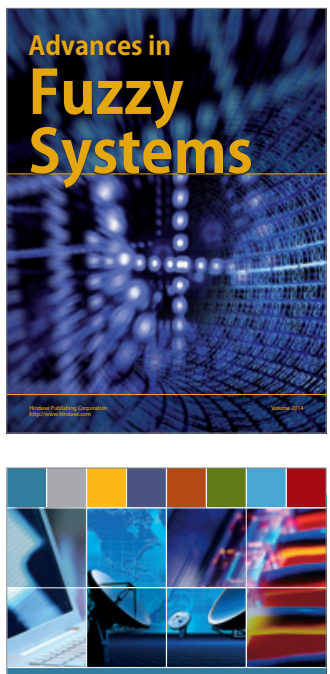

Computer Networks and Communications
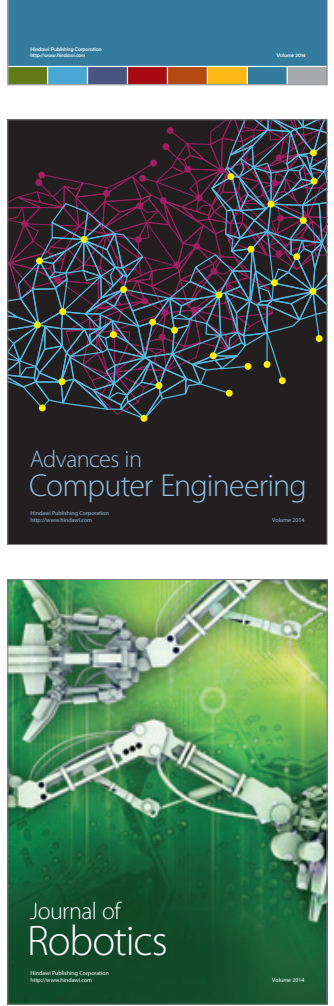
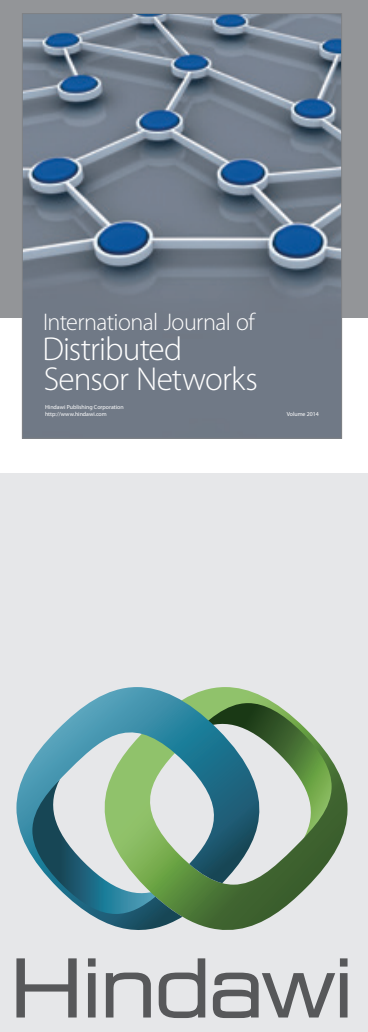

Submit your manuscripts at

http://www.hindawi.com
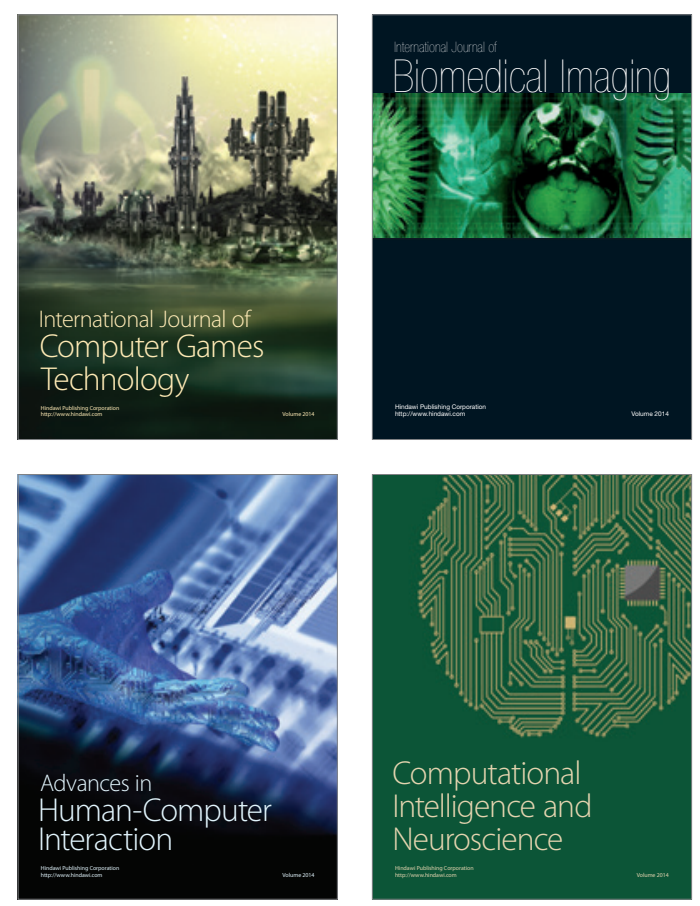
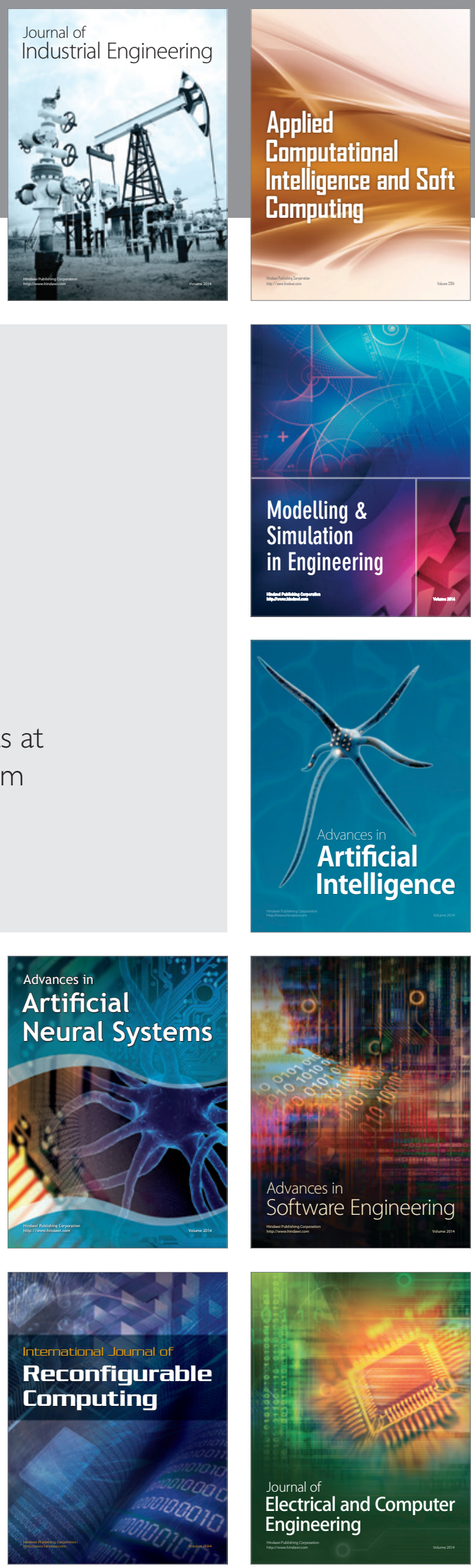